\title{
GRADIENT ESTIMATE ON CONVEX DOMAINS AND APPLICATIONS
}

\author{
FENG-YU WANG AND LIXIN YAN
}

(Communicated by Marius Junge)

\begin{abstract}
By solving the Skorokhod equation for reflecting diffusion processes on a convex domain, gradient estimates for the associated Neumann semigroup are derived. As applications, functional/Harnack inequalities are established for the Neumann semigroup. When the domain is bounded, the gradient estimates are applied to the study of Riesz transforms and regularity of the inhomogeneous Neumann problems on convex domains.
\end{abstract}

\section{INTRODUCTION}

The main purpose of this paper is to study gradient estimates and applications of the Neumann semigroup on a domain $\Omega$ in $\mathbb{R}^{d}$. When the domain is $C^{2}$, the topic has been well studied by using both stochastic analysis (cf. [18, 27, 28, 29]) and an analytic approach (cf. [19, 31, 30]). Both arguments need conditions on the curvature and the second fundamental form of the boundary, since when the boundary is merely Lipschitzian, the second fundamental form is no longer available; thus known results in the above-mentioned references do not apply.

In this paper we adopt a probabilistic approach by solving the Skorokhod equation for the reflecting diffusion process on convex domains. By using the Itô formula for the constructed reflecting diffusion process and a weak dissipative condition, we establish the $L^{1}$ and $L^{2}$ gradient estimates of the corresponding Neumann semigroup.

Let $\Omega \subset \mathbb{R}^{d}$ be a closed domain and let

$$
L=\frac{1}{2} \phi^{2} \Delta+Z \cdot \nabla,
$$

where $\phi \in C^{1}(\Omega)$ is bounded with $\inf _{\Omega} \phi^{2}>0$, and $Z \in C^{1}\left(\Omega ; \mathbb{R}^{d}\right)$ with $|Z(x)| \leq$ $c(1+|x|)$ for some constant $c>0$. We assume that

$$
d|\phi(x)-\phi(y)|^{2}+2\langle Z(x)-Z(y), x-y\rangle \leq K|x-y|^{2}, \quad x, y \in \Omega
$$

holds for some constant $K \in \mathbb{R}$.

Received by the editors January 23, 2011 and, in revised form, July 16, 2011.

2010 Mathematics Subject Classification. Primary 60J75, 60J45; Secondary 42B35, 42B20, $35 \mathrm{~J} 25$.

Key words and phrases. Gradient estimates for Neumann semigroup, reflecting diffusion process, Neumann problem, Hardy space, Green operator.

The first author is supported by SRFDP, the Fundamental Research Funds for the Central Universities and NNSF of China (Grant No. 11131003).

The second author is supported by NNSF of China (Grant No. 10925106), Guangdong Province Key Laboratory of Computational Science and the Fundamental Research Funds for the Central Universities (Grant No. 09lgzs610). 
Let $N$ be the inward pointing unit normal vector field of $\partial \Omega$, the boundary of $\Omega$. For any $f \in \mathcal{B}_{b}(\Omega)$, the set of all bounded measurable functions on $\Omega$, consider the Neumann problem on $\Omega$ :

$$
\left\{\begin{array}{l}
\partial_{t} u(t, \cdot)=L u(t, \cdot),\left.N u(t, \cdot)\right|_{\partial \Omega}=0, \quad t>0 \\
u(0, \cdot)=f .
\end{array}\right.
$$

To characterize the solution of (1.2), we first investigate the reflecting diffusion process generated by $L$ on $\Omega$. Let $B_{t}$ be the $d$-dimensional Brownian motion on a complete filtered probability space with probability measure $\mathbb{P}$.

Theorem 1.1. Let $\Omega$ be convex. For any $x \in \Omega$, there exists a unique adapted continuous process $\left(X_{t}, l_{t}\right)_{t \geq 0}$ on $\Omega \times[0, \infty)$ such that $l_{t}$ is an increasing process with $l_{0}=0$ which increases only when $X_{t} \in \partial \Omega$, and

$$
X_{t}=x+\int_{0}^{t} \phi\left(X_{s}\right)^{2} \mathrm{~d} B_{s}+\int_{0}^{t} Z\left(X_{s}\right) \mathrm{d} s+\int_{0}^{t} N\left(X_{s}\right) \mathrm{d} l_{s}, t \geq 0
$$

holds a.s.

The solution to (1.3) is known as the reflecting diffusion process generated by $L$ on $\Omega$. To indicate the dependence on the initial data $x$, we shall also denote the solution to (1.3) by $\left(X_{t}^{x}, l_{t}^{x}\right)$. By the uniqueness condition we conclude that $X_{t}^{x}$ is a strong Markov process on $\Omega$ with transition semigroup $P_{t}$ given by

$$
P_{t} f(x):=\mathbb{E} f\left(X_{t}^{x}\right), \quad t \geq 0, x \in \Omega, f \in \mathcal{B}_{b}(\Omega) .
$$

It is easy to see by the Itô formula that a smooth solution to (1.2) has to satisfy

$$
u(t, x)=P_{t} f(x), \quad t \geq 0, x \in \Omega .
$$

Thus, $P_{t}$ is nothing but the Neumann semigroup generated by $L$ on $\Omega$.

Due to Theorem 1.1 and a coupling argument, the weak dissipative condition (1.1) implies the following gradient estimates on $P_{t}$.

Theorem 1.2. Let $\Omega$ be convex. For any $f \in C_{b}^{1}(\Omega)$,

$$
\left|\nabla P_{t} f\right|^{2} \leq \mathrm{e}^{K t} P_{t}|\nabla f|^{2}, \quad t \geq 0 .
$$

If moreover $\phi$ is constant, then

$$
\left|\nabla P_{t} f\right| \leq \mathrm{e}^{K t / 2} P_{t}|\nabla f|, \quad t \geq 0 .
$$

To derive functional inequalities for $P_{t}$ using the argument of Bakry and Emery [4, we need the following Kolmogorov equation:

$$
\frac{\mathrm{d}}{\mathrm{d} t} P_{t} f=P_{s} L P_{t-s} f, \quad s \in[0, t], f \in C_{0}^{2}(\Omega),\left.N f\right|_{\partial \Omega}=0 .
$$

When $s=t$ the equation follows from the Itô formula directly. In general, if $P_{t-s} f$ is $\left(C^{2}\right)$ smooth, then the Itô formula for the process $\left\{P_{t-s} f\left(X_{r}\right)\right\}_{r \geq 0}$ implies the desired equation for this $s$. If in particular

$$
Z=\frac{1}{2}\left(\nabla \varphi^{2}+\varphi^{2} \nabla V\right)
$$

holds for some bounded $V \in C^{2}(\Omega)$, then $P_{t}$ is symmetric in $L^{2}(\Omega ; \mu)$ for $\mu(\mathrm{d} x):=$ $\mathrm{e}^{V(x)} \mathrm{d} x$. In this case the generator $(L, \mathcal{D}(L))$ is a negatively definite self-adjoint operator, and for any $t>0$ and $f \in C_{0}(\Omega)$ one has $P_{t} f \in \bigcap_{n \geq 1} \mathcal{D}\left(L^{n}\right)$; that is, $P_{t} f$ is $C^{\infty}$-smooth. 
As consequences of the above gradient estimates, we have the following Poincaré/ log-Sobolev type inequalities and a sharp uniform gradient estimate of $P_{t}$.

Corollary 1.3. Let $\Omega$ be convex and (1.6) hold. Then:

(1) $P_{t} f^{2}-\left(P_{t} f\right)^{2} \leq \frac{\|\phi\|_{\infty}^{2}\left(\mathrm{e}^{K t}-1\right)}{K} P_{t}|\nabla f|^{2}, \quad f \in C_{b}^{1}(\Omega), t \geq 0$.

(2) $\left|\nabla P_{t} f\right|^{2} \leq \frac{K}{\left(\inf \phi^{2}\right)\left(1-\mathrm{e}^{-K t}\right)}\left\{P_{t} f^{2}-\left(P_{t} f\right)^{2}\right\}, \quad f \in \mathcal{B}_{b}(\Omega), t>0$.

(3) If $\phi$ is constant, then

$$
\begin{aligned}
& P_{t} f^{2} \log f^{2}-\left(P_{t} f^{2}\right) \log P_{t} f^{2} \leq \frac{2 \phi^{2}\left(\mathrm{e}^{K t}-1\right)}{K} P_{t}|\nabla f|^{2}, \quad f \in C_{b}^{1}(\Omega), t \geq 0, \\
& \left|\nabla P_{t} f^{2}\right|^{2} \leq \frac{2 K}{\left(1-\mathrm{e}^{-K t}\right) \phi^{2}}\left\{P_{t} f^{2} \log f^{2}-\left(P_{t} f^{2}\right) \log P_{t} f^{2}\right\} P_{t} f^{2}, \quad f \in \mathcal{B}_{b}(\Omega), t>0 .
\end{aligned}
$$

(4) If (1.7) holds for some $V \in C_{b}^{2}(\Omega)$, then there exists a constant $C>0$ independent of $t$ such that

$$
\left\|\nabla P_{t}\right\|_{1 \rightarrow \infty} \leq \frac{C}{(1 \wedge t)^{(d+1) / 2}}
$$

holds, where $\|\cdot\|_{1 \rightarrow \infty}$ is the operator norm from $L^{1}(\Omega ; \mu)$ to $L^{\infty}(\Omega ; \mu)$.

Finally, according to [24, Proof of Theorem 2.1] and [26. Proof of Lemma 2.1], the gradient estimates (1.4) and (1.5) imply the following Harnack type inequalities (1.8) and (1.9) respectively. These Harnack inequalities have a number of applications to heat kernel estimates and entropy-cost inequalities, for which we refer to e.g. [14, 24, 10].

Corollary 1.4. In the situation of Corollary 1.3, let $t>0$ be fixed. Then for any $f \in \mathcal{B}_{b}(\Omega)$ with $f \geq 1$,

$$
P_{t} \log f(x) \leq \log P_{t} f(y)+\frac{K|x-y|^{2}}{4\left(\inf \phi^{2}\right)\left(1-\mathrm{e}^{-K t}\right)}, \quad x, y \in \Omega
$$

holds. If $\phi$ is constant, then for any $p>1$ and positive $f \in \mathcal{B}_{b}(\Omega)$,

$$
\left(P_{t} f\right)^{p}(x) \leq\left(P_{t} f^{p}(y)\right) \exp \left[\frac{K p|x-y|^{2}}{4(p-1) \phi^{2}\left(1-\mathrm{e}^{-K t}\right)}\right], \quad x, y \in \Omega
$$

holds.

The proofs of Theorems 1.1, 1.2 and Corollary 1.3 will be given in Section 2. In Section 3, we introduce an application of our results to the study of Riesz transforms and regularity of the inhomogeneous Neumann problems on convex domains following the line of work in [12].

\section{ProOFs}

2.1. Proof of Theorem 1.1. By the convexity of $\Omega$ and the weak dissipative condition (1.1), it is easy to see that for every $x \in \Omega$, the equation (1.3) has at most one solution. Indeed, let $\left(X_{t}, l_{t}\right)$ and $\left(\tilde{X}_{t}, \tilde{l}_{t}\right)$ be solutions of (1.3). Since $l_{t}$ and $\tilde{l}_{t}$ are local times of $X_{t}$ and $\tilde{X}_{t}$ on $\partial \Omega$ respectively, it suffices to show that $X_{t}=\tilde{X}_{t}$. Since $\Omega$ is convex,

$$
\langle y-z, N(y)\rangle \leq 0, \quad y \in \partial \Omega, z \in \Omega .
$$


Noting that $l_{t}$ (resp. $\left.\tilde{l}_{t}\right)$ increases only when $X_{t} \in \partial \Omega$ (resp. $\tilde{X}_{t} \in \partial \Omega$ ), this implies that

$$
\left\langle X_{t}-\tilde{X}_{t}, N\left(X_{t}\right) \mathrm{d} l_{t}-N\left(\tilde{X}_{t}\right) \mathrm{d} \tilde{l}_{t}\right\rangle \leq 0 .
$$

Combining this with the Itô formula and (1.1), we obtain

$$
\begin{aligned}
\mathrm{d}\left|X_{t}-\tilde{X}_{t}\right|^{2}=\{ & \left.2\left\langle X_{t}-\tilde{X}_{t}, Z\left(X_{t}\right)-Z\left(\tilde{X}_{t}\right)\right\rangle+d\left|\phi\left(X_{t}\right)-\phi\left(\tilde{X}_{t}\right)\right|^{2}\right\} \mathrm{d} t \\
& +2\left\langle\left(\phi\left(X_{t}\right)-\phi\left(\tilde{X}_{t}\right)\right) \mathrm{d} B_{t}, X_{t}-\tilde{X}_{t}\right\rangle \\
\leq & K\left|X_{t}-\tilde{X}_{t}\right|^{2} \mathrm{~d} t+2\left\langle\left(\phi\left(X_{t}\right)-\phi\left(\tilde{X}_{t}\right)\right) \mathrm{d} B_{t}, X_{t}-\tilde{X}_{t}\right\rangle .
\end{aligned}
$$

This implies that

$$
\mathbb{E}\left|X_{t}-\tilde{X}_{t}\right|^{2} \leq\left|X_{0}-\tilde{X}_{0}\right|^{2} \mathrm{e}^{K t}=0, \quad t \geq 0 .
$$

As soon as the uniqueness is confirmed, by the Yamada-Watanabe principle [32, it remains to prove the existence of a weak solution. We shall complete the proof by using known results for the reflecting Brownian motion on compact convex domains, an approximation argument, the Girsanov theorem and a time-change argument. Although the idea of the proof is rather fundamental in stochastic analysis, we did not find any reference which explicitly includes the following details for SDEs with reflection on a non-smooth domain.

(a) Let $\Omega$ be compact and $L=\frac{1}{2} \Delta$. By using the regularity of the associated Dirichlet form, [6. Theorem 4.4] ensures the existence of a reflecting Brownian motion. According to [5, Theorem 1], there exists a $d$-dimensional Brownian motion $\tilde{W}_{t}$ and a continuous adapted process $\left(\tilde{X}_{t}, \tilde{l}_{t}\right)$ on $\Omega \times[0, \infty)$ such that $\tilde{l}_{t}$ is an increasing process with $\tilde{l}_{0}=0$ which increases only when $\tilde{X}_{t} \in \partial \Omega$, and

$$
\tilde{X}_{t}=x+\tilde{W}_{t}+\int_{0}^{t} N\left(\tilde{X}_{s}\right) \mathrm{d} \tilde{l}_{s}, \quad t \geq 0
$$

holds.

(b) Let $\Omega$ be non-compact and $L=\frac{1}{2} \Delta$. In this case we may construct the solution by a simple approximation argument. Let

$$
\Omega_{n}=\Omega \cap\{z:|z-x| \leq n\}, \quad n \geq 1 .
$$

Then for each $n, \Omega_{n}$ is a compact convex domain. By (a) and the Yamada-Watanabe principle, there exists a unique solution to the equation

$$
X_{t}^{(n)}=x+B_{t}+\int_{0}^{t} N^{(n)}\left(X_{s}^{(n)}\right) \mathrm{d} l_{s}^{(n)}, \quad t \geq 0,
$$

where $N^{(n)}$ is the inward pointing unit vector field of $\partial \Omega_{n}$. Let

$$
\tau_{n}=\inf \left\{t \geq 0:\left|X_{t}^{(n)}-x\right| \geq n\right\} .
$$

By the uniqueness of a strong solution to (2.3), we have $\left(X_{t}^{(n)}, l_{t}^{(n)}\right)=\left(X_{t}^{(m)}, l_{t}^{(m)}\right)$ for $t \leq \tau_{n} \wedge \tau_{m}$. In particular, $\tau_{n}$ is increasing in $n$. By the convexity of the boundary and the Itô formula, we obtain from (2.3) that

$$
\left|X_{t}^{(n)}-x\right|^{2} \leq t d+2 \int_{0}^{t}\left\langle X_{s}^{(n)}, \mathrm{d} B_{s}\right\rangle, \quad t \geq 0
$$

This implies that

$$
n \mathbb{P}\left(\tau_{n} \leq t\right) \leq \mathbb{E}\left|X_{t \wedge \tau_{n}}-x\right|^{2} \leq t d
$$


Therefore, $\tau_{n} \uparrow \infty$ as $n \uparrow \infty$. This enables us to observe that

$$
\left(X_{t}, l_{t}\right):=\sum_{n=1}^{\infty}\left(X_{t}^{(n)}, l_{t}^{(n)}\right) 1_{\left[\tau_{n-1}, \tau_{n}\right)}, \quad \tau_{0}:=0
$$

solves the equation

$$
X_{t}=x+B_{t}+\int_{0}^{t} N\left(X_{s}\right) \mathrm{d} l_{s}, \quad t \geq 0 .
$$

(c) Let $\left(X_{t}, l_{t}\right)$ solve (2.4). Then

$$
X_{t}=x+\tilde{B}_{t}+\int_{0}^{t} Z\left(X_{s}\right) \mathrm{d} s+\int_{0}^{t} N\left(X_{s}\right) \mathrm{d} B_{s}, \quad t \geq 0
$$

holds for

$$
\tilde{B}_{t}:=B_{t}-\int_{0}^{t} Z\left(X_{s}\right) \mathrm{d} s, \quad t \geq 0
$$

Since $Z$ has linear growth, it is easy to see that

$$
t \mapsto R_{t}:=\exp \left[\int_{0}^{t}\left\langle Z\left(X_{s}\right), \mathrm{d} B_{s}\right\rangle-\frac{1}{2} \int_{0}^{t}\left|Z\left(X_{s}\right)\right|^{2} \mathrm{~d} s\right]
$$

is a martingale. By the Girsanov theorem, for any $T>0$ the process $\left\{\tilde{B}_{t}\right\}_{t \leq T}$ is a $d$-dimensional Brownian motion under the probability $R_{T} \mathbb{P}$. Therefore, under the probability measure $R_{T} \mathbb{P},\left(X_{t}, l_{t}\right)_{t \leq T}$ is a weak solution of (1.3) for $\phi \equiv 1$. By the Yamada-Watanabe principle, this equation has a unique strong solution up to time $T$. This proves the desired assertion for $\phi \equiv 1$ since $T>0$ is arbitrary.

(d) Since inf $\phi^{2}>0$ and $Z$ has linear growth, $\frac{Z}{\phi^{2}}$ has linear growth as well. By (c), let $\left(X_{t}, l_{t}\right)$ solve the equation

$$
X_{t}=x+B_{t}+\int_{0}^{t} \frac{Z\left(X_{s}\right)}{\phi\left(X_{s}\right)^{2}} \mathrm{~d} s+\int_{0}^{t} N\left(X_{s}\right) \mathrm{d} l_{s}, \quad t \geq 0 .
$$

Let

$$
\tau(t)=\int_{0}^{t} \frac{\mathrm{d} s}{\phi\left(X_{s}\right)^{2}}, \sigma(t)=\tau^{-1}(t), \quad t \geq 0 .
$$

Since $\inf \phi^{2}>0$ and $\phi$ is bounded, there exists a constant $C>1$ such that $\frac{t}{C} \leq$ $\sigma(t) \leq C t, t \geq 0$. Obviously,

$$
\sigma^{\prime}(t)=\phi\left(X_{\sigma(t)}\right)^{2}, \quad t \geq 0 .
$$

Thus,

$$
\int_{0}^{\sigma(t)} \frac{Z\left(X_{s}\right)}{\phi\left(X_{s}\right)^{2}} \mathrm{~d} s=\int_{0}^{t} \frac{Z\left(X_{\sigma(s)}\right)}{\phi\left(X_{\sigma(s)}\right)^{2}} \mathrm{~d} \sigma(s)=\int_{0}^{t} Z\left(X_{\sigma(s)}\right) \mathrm{d} s .
$$

Moreover, let $\tilde{l}_{s}=l_{\sigma(s)}$, which is a continuous increasing process with support included in $\left\{s \geq 0: X_{\sigma(s)} \in \partial \Omega\right\}$. We have

$$
\int_{0}^{\sigma(t)} N\left(X_{s}\right) \mathrm{d} l_{s}=\int_{0}^{t} N\left(X_{\sigma(s)}\right) \mathrm{d} \tilde{l}_{s}, \quad t \geq 0 .
$$

Finally, let

$$
\tilde{B}_{t}=\int_{0}^{t} \frac{1}{\phi\left(X_{\sigma(s)}\right)} \mathrm{d} B_{\sigma(s)}, \quad t \geq 0 .
$$


We have

$$
B_{\sigma(t)}=\int_{0}^{t} \phi\left(X_{\sigma(s)}\right) \mathrm{d} \tilde{B}_{s}, \quad t \geq 0 .
$$

Since $\tilde{B}_{t}$ is a continuous martingale with quadratic process

$$
\langle\tilde{B}\rangle_{t}=\int_{0}^{t} \frac{1}{\phi\left(X_{\sigma(s)}\right)^{2}} \mathrm{~d}\langle B\rangle_{\sigma(s)}=\int_{0}^{t} \frac{\mathrm{d} \sigma(s)}{\phi\left(X_{\sigma(s)}\right)^{2}}=t,
$$

it is indeed a Brownian motion. Therefore, combining (2.5), (2.6), (2.7) and (2.8), we conclude that $\left(\tilde{X}_{t}, \tilde{l}_{t}\right):=\left(X_{\sigma(t)}, l_{\sigma(t)}\right)$ is a weak solution of (1.3).

\subsection{Proofs of Theorem $\mathbf{1 . 2}$ and Corollary $\mathbf{1 . 3}$,}

Proof of Theorem 1.2. For any $x \in \Omega$, let $\left(X_{t}^{x}, l_{t}^{x}\right)$ be the unique solution to (1.3) ensured by Theorem 1.1. As explained in the beginning of subsection 2.1, by (1.1), (2.1) and using the Itô formula, we obtain

$$
\mathrm{d}\left|X_{t}^{x}-X_{t}^{y}\right|^{2} \leq K\left|X_{t}^{x}-X_{t}^{y}\right|^{2} \mathrm{~d} t+2\left\langle X_{t}^{x}-X_{t}^{y},\left(\phi\left(X_{t}^{x}\right)-\phi\left(X_{t}^{y}\right)\right) \mathrm{d} B_{t}\right\rangle .
$$

This implies that

$$
\mathbb{E}\left|X_{t}^{x}-X_{t}^{y}\right|^{2} \leq|x-y|^{2} \mathrm{e}^{K t}, \quad t \geq 0, x, y \in \Omega .
$$

Therefore,

$$
\begin{aligned}
& \left|\nabla P_{t} f(x)\right|:=\limsup _{y \rightarrow x} \frac{\left|P_{t} f(y)-P_{t} f(x)\right|}{|x-y|} \leq \limsup _{y \rightarrow x} \frac{\mathbb{E}\left|f\left(X_{t}^{x}\right)-f\left(X_{t}^{y}\right)\right|}{|x-y|} \\
& \quad \leq \limsup _{y \rightarrow x}\left(\mathbb{E} \frac{\left|f\left(X_{t}^{x}\right)-f\left(X_{t}^{y}\right)\right|^{2}}{\left|X_{t}^{x}-X_{t}^{y}\right|^{2}}\right)^{1 / 2}\left(\frac{\mathbb{E}\left|X_{t}^{x}-X_{t}^{y}\right|^{2}}{|x-y|^{2}}\right)^{1 / 2}=\left(P_{t}|\nabla f|^{2}\right)^{1 / 2} \mathrm{e}^{K t / 2} .
\end{aligned}
$$

This proves (1.4).

If $\phi$ is constant, then the martingale term in (2.9) disappears, so that

$$
\left|X_{t}^{x}-X_{t}^{y}\right| \leq|x-y| \mathrm{e}^{K t / 2}, \quad t \geq 0
$$

holds. Therefore,

$$
\begin{aligned}
\left|\nabla P_{t} f(x)\right| & :=\limsup _{y \rightarrow x} \frac{\left|P_{t} f(y)-P_{t} f(x)\right|}{|x-y|} \\
& \leq \mathrm{e}^{K t / 2} \limsup _{y \rightarrow x} \frac{\mathbb{E}\left|f\left(X_{t}^{x}\right)-f\left(X_{t}^{y}\right)\right|}{\left|X_{t}^{x}-Y_{t}^{y}\right|}=\mathrm{e}^{K t / 2} P_{t}|\nabla f| .
\end{aligned}
$$

Proof of Corollary 1.3, Let $t>0$ and $f \in C_{0}^{\infty}(\Omega)$. By (1.4) and (1.6),

$$
\frac{\mathrm{d}}{\mathrm{d} s} P_{s}\left(P_{t-s} f\right)^{2}=P_{s}\left\{\phi^{2}\left|\nabla P_{t-s} f\right|^{2}\right\} \geq\left(\inf \phi^{2}\right) \mathrm{e}^{K s}\left|\nabla P_{t} f\right|^{2}
$$

holds for $s \in[0, t]$. Integrating both sides over $[0, t]$, we prove (2). Moreover, (1.4) also implies that

$$
P_{s}\left\{\phi^{2}\left|\nabla P_{t-s} f\right|^{2}\right\} \leq \mathrm{e}^{(t-s) K}\|\phi\|_{\infty}^{2} P_{t}|\nabla f|^{2} .
$$

Combining this with the first equality in (2.10) we prove (1).

When $\phi$ is constant, (3) can be proved in the same way by using (1.5) in place of (1.4) and by considering

$$
\frac{\mathrm{d}}{\mathrm{d} s} P_{s}\left\{\left(P_{t-s} f^{2}\right) \log P_{t-s} f^{2}\right\} .
$$


Finally, if $Z_{i}$ is given in (4), then $P_{t}$ is symmetric in $L^{2}(\Omega ; \mu)$. Since $\Omega$ is convex, the classical Nash inequality is implied by [11, Theorem 3.2.9]; i.e., there exists a constant $C>0$ such that

$$
\int_{\Omega} f(x)^{2} \mathrm{~d} x \leq C+C\left(\int_{\Omega}|\nabla f|^{2}(x) \mathrm{d} x\right)^{d /(d+2)}, \quad f \in W^{2,1}(\Omega ; \mathrm{d} x), \int_{\Omega}|f|(x) \mathrm{d} x=1 .
$$

Since $V$ is bounded, the same inequality holds for $\mu(\mathrm{d} x)$ in place of $\mathrm{d} x$ for some different constant $C>0$. Therefore,

$$
\left\|P_{t}\right\|_{1 \rightarrow \infty} \leq \frac{c}{(1 \wedge t)^{d / 2}}, \quad t>0
$$

holds for some constant $c>0$. Combining this with (2) for $P_{t} f$ in place of $f$, we prove (4) immediately.

\section{Applications: Riesz transforms and Regularity of the INHOMOGENEOUS NEUMANN PROBLEMS ON CONVEX DOMAINS}

Throughout this section, we let $\Omega$ be a bounded convex domain in $\mathbb{R}^{d}$, and let

$$
L=\frac{1}{2} \varphi^{2} \Delta+Z \cdot \nabla \quad \text { such that } Z \text { is given in (1.7). }
$$

Thus, the generator $(L, \mathcal{D}(L))$ of $P_{t}$ in $L^{2}(\Omega ; \mu)$ is a negatively definite selfadjoint operator with discrete spectrum. Let $\mathcal{L}_{N}=-L$, which is thus a positive definite self-adjoint operator such that $P_{t}=\mathrm{e}^{-\mathcal{L}_{N} t}, t \geq 0$.

It is well-known that (2.11) self-improves into the off-diagonal upper estimates; i.e., there exists a constant $c_{1}>0$ such that the kernel $p_{t}(x, y)$ of $P_{t}$ satisfies

$$
\left|p_{t}(x, y)\right| \leq \frac{C}{\left|B^{\Omega}(x, \sqrt{t})\right|} \exp \left(-\frac{|x-y|^{2}}{c_{1} t}\right), \quad t>0, x, y \in \Omega .
$$

See, for example, Theorem 1.1 of $[15$. Here, $|E|$ denotes the Lebesgue measure of a measurable set $E \subseteq \mathbb{R}^{d}$, and for $x \in \mathbb{R}^{d}$ and $r>0$ we have set $B^{\Omega}(x, r):=\{y \in$ $\Omega:|x-y|<r\}$.

Lemma 3.1. Let $\Omega$ be a bounded convex domain in $\mathbb{R}^{d}$. Then there exist constants $C, c_{2}, c_{3}>0$ such that the kernel $p_{t}(x, y)$ of $P_{t}$ satisfies

$$
\left|\nabla p_{t}(x, y)\right| \leq\left\{\begin{array}{l}
C t^{-(d+1) / 2} \exp \left(-\frac{|x-y|^{2}}{c_{2} t}\right), \quad 0<t \leq 1 \\
C e^{-c_{3} t} \exp \left(-\frac{|x-y|^{2}}{c_{2} t}\right), \quad t>1
\end{array}\right.
$$

for all $x, y \in \Omega$.

Proof. Using $\frac{\mu}{\mu(\Omega)}$ to replace $\mu$, we may assume that $\mu$ is a probability measure. From the self-improvement property (see, for example, Theorem 4.9 of [9]), it suffices to show that there exist constants $C, c_{4}>0$ such that

$$
\left|\nabla p_{t}(x, y)\right| \leq\left\{\begin{array}{l}
C t^{-(d+1) / 2}, \quad 0<t \leq 2 \\
C e^{-c_{4} t}, \quad t>2
\end{array}\right.
$$

for all $x, y \in \Omega$. 
Indeed, we observe that from Corollary [1.3(4), there exists a constant $C_{1}>0$ such that

$$
\left\|\nabla P_{t}\right\|_{1 \rightarrow \infty} \leq \frac{C_{1}}{(1 \wedge t)^{(d+1) / 2}}, \quad t>0 .
$$

On the other hand, since $\Omega$ is compact, $\mathcal{L}_{N}$ has a spectral gap $\lambda_{1}(>0)$, which is known as the first Neumann eigenvalue of $\mathcal{L}_{N}$ on $\Omega$. We have

$$
\mu\left(\left|P_{t} \hat{f}\right|^{2}\right) \leq \mathrm{e}^{-\lambda_{1} t} \mu\left(|\hat{f}|^{2}\right), \quad t \geq 0, f \in L^{2}(\Omega ; \mu),
$$

where $\hat{f}:=f-\mu(f)$. Therefore, for any $t>2$ and $f \in L^{1}(\Omega ; \mu)$ with $\|f\|_{1} \leq 1$,

$$
\begin{aligned}
\left\|\nabla P_{t} f\right\|_{1 \rightarrow \infty} & =\left\|\nabla P_{1}\left(P_{t-1} \hat{f}\right)\right\|_{\infty} \leq\left\|P_{1}\right\|_{1 \rightarrow \infty}\left\|P_{t-1} \hat{f}\right\|_{1} \\
& \leq C_{1}\left\|P_{t-2}\left(P_{1} \hat{f}\right)\right\|_{2} \leq C_{1} \mathrm{e}^{-\lambda_{1} t}\left\|P_{1} \hat{f}\right\|_{2} \leq 2 C_{1}\left\|P_{1}\right\|_{1 \rightarrow 2} \mathrm{e}^{-\lambda_{1} t} .
\end{aligned}
$$

Since due to (2.11) one has $\left\|P_{1}\right\|_{1 \rightarrow 2}<\infty$, estimate (3.4) follows readily, and the proof of Lemma 3.1 is finished.

Before continuing we need to clarify some notation. Recall that $\mu \mathrm{d} x=e^{V(x)} \mathrm{d} x$. Since $V \in C^{2}(\Omega)$ is bounded, we have that $L^{p}(\Omega ; \mu)=L^{p}(\Omega ; \mathrm{d} x)$. In the next two sections, we do not have to distinguish integrals w.r.t. $\mu \mathrm{d} x$ and $\mathrm{d} x$, except if the self-adjointness and integration by parts formula are concerned.

3.1. Riesz transforms on bounded convex domains. Since $\phi \in C^{1}(\Omega)$ is bounded with $\inf _{\Omega} \phi^{2}>0$, and $V \in C^{2}(\Omega)$ is bounded, we have that for every $f \in L^{2}(\Omega)$,

$$
\begin{aligned}
\int_{\Omega}\left|\nabla \mathcal{L}_{N}^{-1 / 2} f(x)\right|^{2} \mathrm{~d} x & \leq C\left(\inf _{\Omega} \varphi^{2}\right)^{-1} \int_{\Omega} \varphi^{2} \nabla \mathcal{L}_{N}^{-1 / 2} f(x) \overline{\nabla \mathcal{L}_{N}^{-1 / 2} f(x)} \mu \mathrm{d} x \\
& \leq C Q\left(\mathcal{L}_{N}^{-1 / 2} f, \mathcal{L}_{N}^{-1 / 2} f\right) \\
& =C \int_{\Omega}|f(x)|^{2} \mu \mathrm{d} x \\
& \leq C\|f\|_{L^{2}(\Omega)}^{2}
\end{aligned}
$$

where

$$
Q(u, v):=\frac{1}{2} \int_{\Omega} \varphi^{2} \nabla u \overline{\nabla v} \mu \mathrm{d} x, \quad u, v \in W^{1,2}(\Omega),
$$

and the latter equality follows from the fact that $Q$ is symmetric. Hence $\mathcal{D}(Q)=$ $\mathcal{D}\left(\mathcal{L}_{N}^{1 / 2}\right)$ and $Q(u, v)=\left(\mathcal{L}_{N}^{1 / 2} u, \mathcal{L}_{N}^{1 / 2} v\right)$ (see, for example, Theorem 8.1 of [23]). Therefore, the Riesz transform $T=\nabla \mathcal{L}_{N}^{-1 / 2}$ is bounded on $L^{2}(\Omega)$.

In terms of the gradient estimate (3.3) of the kernel of the semigroup $P_{t}, t>0$, we have the following result. For its proof, it is almost identical to that of Theorem 1.4 of [3] and is omitted here.

Theorem 3.2. Let $\Omega$ be a bounded convex domain in $\mathbb{R}^{d}$. Then the Riesz transform $T=\nabla \mathcal{L}_{N}^{-1 / 2}$ is of weak type $(1,1)$ and bounded on $L^{p}(\Omega)$ for all $1<p<\infty$. That is, there exists $C_{p}, 1 \leq p<\infty$ such that

$$
\left\|\nabla \mathcal{L}_{N}^{-1 / 2}\right\|_{L^{p}(\Omega)} \leq C_{p}\|f\|_{L^{p}(\Omega)}
$$

and

$$
\left\|\nabla \mathcal{L}_{N}^{-1 / 2}\right\|_{L^{1, \infty}(\Omega)} \leq C_{1}\|f\|_{L^{1}(\Omega)} .
$$


3.2. Regularity of the inhomogeneous Neumann problems on convex domains. Given an open, bounded subset $\Omega$ of $\mathbb{R}^{d}$ and $f \in C^{\infty}(\bar{\Omega})$, we define the Neumann Green operator $\mathbb{G}_{N}$ as the solution operator $C^{\infty}(\bar{\Omega}) \ni f \mapsto u=\mathbb{G}_{N}(f) \in$ $W^{1,2}(\Omega)$ for the Neumann problem

$$
\left\{\begin{aligned}
-L u=f & \text { in } \quad \Omega, \\
\partial_{\nu} u=0 & \text { on } \quad \partial \Omega,
\end{aligned}\right.
$$

where it is also assumed that $\int_{\Omega} f \mu \mathrm{d} x=0$. Above, $\nu(x)$ denotes the outward unit normal to $\partial \Omega$ at $x \in \partial \Omega$, and $\partial_{\nu}=\nu \cdot \nabla$ stands for the normal derivative. The main result of this section is:

Theorem 3.3. Let $\Omega$ be a bounded convex domain in $\mathbb{R}^{d}$. Let $\mathbb{G}_{N}$ be the Neumann Green operator for the problem (3.5). Then the operators

$$
\frac{\partial^{2} \mathbb{G}_{N}}{\partial x_{i} \partial x_{j}}, \quad i, j=1, \ldots, n,
$$

originally defined on $\left\{f \in C^{\infty}(\bar{\Omega}): \int_{\Omega} f \mu \mathrm{d} x=0\right\}$, can be extended to bounded operators on $L^{p}(\Omega)$ for $1<p \leq 2$.

If $p=1$, then the operators $\frac{\partial^{2} \mathbb{G}_{N}}{\partial x_{i} \partial x_{j}}, i, j=1, \ldots, n$, are also of weak type $(1,1)$.

The strategy employed in the proof of Theorem 3.3 is to continue the line of study in [12] where X.T. Duong et al. establish regularity results for the Green operator, regarded as the inverse of the Neumann Laplacian on bounded convex domains. Indeed, the kernel of the operator $\nabla^{2} L^{-1}$ is formally given by

$$
\int_{0}^{\infty} \nabla_{x}^{2} p_{t}(x, y) d t
$$

and the second-order derivative $\nabla_{x}^{2} p_{t}(x, y)$ of the kernel $p_{t}(x, y)$, associated with the heat semigroup $e^{-t L}$, does not satisfy a Hölder condition in a spatial variable. The loss of Hölder continuity is compensated by a more subtle built-in regularity property inherited from the semigroup $e^{-t L}$, and by the availability of certain regularity estimates of the solution to the Neumann problem which is specific to convex domains. For more information about the background of this topic, we refer the reader to [1, 2, 12, 17, 16, 20, 22, 21] and the references therein.

3.2.1. $L^{2}$-estimate. The following coercive estimates in convex domains are going to play a basic role in subsequent developments, whose proof follows the lines of Theorem 3.1.3.3 in [16] and Theorem 2.1 in [1].

Lemma 3.4. Let $\Omega$ be a bounded convex domain in $\mathbb{R}^{d}$. Let $f \in L^{2}(\Omega)$ have bounded support, and let $u$ be the unique function in $W^{1,2}(\Omega)$ satisfying (3.5). Then $u \in W^{2,2}(\Omega)$ and for any $\psi \in C^{\infty}\left(\mathbb{R}^{d}\right)$ it follows that

$$
\int_{\Omega} \psi^{2}\left|\nabla^{2} u\right|^{2} \mathrm{~d} x \leq C \int_{\Omega}\left(|\nabla \psi|^{2}+\psi^{2}\right)|\nabla u|^{2} \mathrm{~d} x+C \int_{\Omega} \psi^{2} f^{2} \mathrm{~d} x
$$

for some constant $C>0$, depending on $\varphi$ and $V$ of $L$, not depending on $\psi$. Moreover, one also has

$$
\int_{\Omega}\left|\nabla^{2} u\right|^{2} \mathrm{~d} x \leq C \int_{\Omega}|\nabla u|^{2} \mathrm{~d} x+C \int_{\Omega} f^{2} \mathrm{~d} x .
$$


Proof. Begin by assuming that $\partial \Omega$ and $f$ are $C^{\infty}$. Kadlec's formula on p. 134 of [16] implies that

$$
\int_{\Omega}\left(\operatorname{div}\left(\psi \varphi^{2} e^{V} \nabla u\right)\right)^{2} \mathrm{~d} x=b+\int_{\Omega} \sum_{i, j}\left(\psi \varphi^{2} e^{V} \nabla u_{i}\right)_{j}\left(\psi \varphi^{2} e^{V} \nabla u_{j}\right)_{i} \mathrm{~d} x
$$

where $b$ is a boundary integral, and the subscripts $i$ and $j$ denote derivatives with respect to the coordinates $x_{i}$ and $x_{j}$. Because $\nu \cdot\left(\psi \varphi^{2} e^{V} \nabla u\right)=\psi \varphi^{2} e^{V} \partial_{\nu} u=0$ on $\partial \Omega, b$ is the integral over the boundary of the second fundamental form evaluated at the tangential component of $\psi \varphi^{2} e^{V} \nabla u$. The only thing we need to know about $b$ is that it is non-negative. Hence,

$$
\begin{gathered}
\int_{\Omega} \sum_{i, j}\left(\psi^{2} \varphi^{4} e^{2 V} u_{i j}^{2}+2\left(\psi \varphi^{2} e^{V}\right)_{j} u_{i}\left(\psi \varphi^{2} e^{V}\right) u_{i j}+\left(\psi \varphi^{2} e^{V}\right)_{i}\left(\psi \varphi^{2} e^{V}\right)_{j} u_{i} u_{j}\right) \mathrm{d} x \\
\leq \int_{\Omega}\left(\psi \operatorname{div}\left(\varphi^{2} e^{V} \nabla u\right)+\nabla \psi \cdot \varphi^{2} e^{V} \nabla u\right)^{2} \mathrm{~d} x
\end{gathered}
$$

Observe that $\operatorname{div}\left(\varphi^{2} e^{V} \nabla u\right)=2 e^{V} L u$ and $\left(\psi \varphi^{2} e^{V}\right)_{i}=\left(\psi_{i} \varphi^{2}+2 \psi Z_{i}\right) e^{V}$. Since $\phi \in C^{1}(\Omega)$ is bounded with $\inf _{\Omega} \phi^{2}>0$, and $Z \in C^{1}\left(\Omega ; \mathbb{R}^{d}\right)$ with $|Z(x)| \leq c(1+|x|)$ for some constant $c>0$, and $V \in C^{2}(\Omega)$ is bounded, we use the inequality $2 x y \leq$ $(1 / C) x^{2}+C y^{2}$ to obtain

$$
\int_{\Omega} \psi^{2}\left|\nabla^{2} u\right|^{2} \mathrm{~d} x \leq C \int_{\Omega}\left(|\nabla \psi|^{2}+|\psi|^{2}\right)|\nabla u|^{2} \mathrm{~d} x+C \int_{\Omega} \psi^{2} f^{2} \mathrm{~d} x
$$

for some constant $C$, depending on $\varphi$ and $V$. Finally, (3.9) follows from (3.8) by taking $\psi \equiv 1$.

A routine limiting argument yields the result for all convex bounded domains $\Omega$. See, for example, p. 149 of [16].

Proposition 3.5. Let $\Omega$ be a bounded convex domain in $\mathbb{R}^{d}$. Let $\mathbb{G}_{N}$ be the Neumann Green operator for the problem (3.5). Then $\nabla^{2} \mathbb{G}_{N} f \in L^{2}(\Omega)$ and

$$
\int_{\Omega}\left|\nabla^{2} \mathbb{G}_{N} f\right|^{2} \mathrm{~d} x \leq \int_{\Omega}|f|^{2} \mathrm{~d} x
$$

for some constant $C>0$ independent of $f$.

Proof. To estimate (3.11), from (3.9) it reduces to showing that there exists a constant $C>0$ independent of $f$ such that

$$
\int_{\Omega}\left|\nabla \mathbb{G}_{N}(f)\right|^{2} \mathrm{~d} x \leq C \int_{\Omega}|f|^{2} \mathrm{~d} x .
$$

Indeed, based on the gradient estimate (3.4) of the heat kernel $p_{t}(x, y)$ in Lemma 3.1 . we have

$$
\left|\nabla \mathbb{G}_{N}(x, y)\right| \leq \frac{C}{|x-y|^{d-1}}, \quad \forall x, y \in \Omega
$$

Estimate (3.12) follows readily. This proves Proposition 3.5 


\subsubsection{Proof of Theorem 3.3 .}

3.2.2.1. Singular integrals. The following theorem is Theorem 1, 13 .

Theorem 3.6. Let $T$ is bounded linear operator from $L^{2}(\Omega)$ into $L^{2}(\Omega)$ with $\Omega$. Assume that there exists a class of operators $A_{t}, t>0$, defined on $L^{2}(\Omega)$, which is represented by kernels $a_{t}(x, y)$ in the sense that

(a) $A_{t} u(x)=\int_{\Omega} a_{t}(x, y) u(y) d y$ for any function $u \in L^{2}(\Omega) \cap L^{1}(\Omega)$, and the kernels $a_{t}(x, y)$ satisfy the following condition:

$$
\left|a_{t}(x, y)\right| \leq \frac{1}{\left|B^{\Omega}(y, \sqrt{t})\right|\left(1+\frac{|x-y|}{\sqrt{t}}\right)^{n+\beta}}, \quad \forall x, y \in \Omega, \forall t>0, \beta>0 .
$$

(b) The composite operator $T\left(I-A_{t}\right)$ has a measurable kernel $\tilde{K}_{t}(x, y)$, and there exist constants $C$ and $c>0$ such that

$$
\int_{x \in \Omega:|x-y| \geq c \sqrt{t}}\left|\tilde{K}_{t}(x, y)\right| d x \leq C, \quad y \in \Omega .
$$

Moreover, the operator $T$ is of weak type $(1,1)$. Hence $T$ can be extended from $L^{2}(\Omega) \cap L^{p}(\Omega)$ to a bounded operator on $L^{p}(\Omega)$ for all $1<p \leq 2$.

3.2.2.2. Estimates for the space derivatives of the heat kernel. Recall that $p_{t}(x, y)$ is the kernel of $e^{-t \mathcal{L}_{N}}$, which satisfies estimate (3.2). Since Gaussian upper bounds carry over from heat kernels to their time derivative of its kernels (see 11] and [23]), we have

$$
\left|\frac{\mathrm{d}}{\mathrm{d} t} p_{t}(x, y)\right| \leq \frac{C}{t^{1 / 2}\left|B^{\Omega}(x, \sqrt{t})\right|} \exp \left(-\frac{|x-y|^{2}}{c_{5} t}\right), \quad t>0, x, y \in \Omega
$$

for some constant $c_{5}>0$. Note that $p_{t}(\cdot, y)$ and their time derivatives $\frac{d}{d t} p_{t}(\cdot, y)$ belong to the domain of $\mathcal{D}\left(\mathcal{L}_{N}\right)$. In particular, $p_{t}(\cdot, y) \in W^{1,2}(\Omega)$. When $\Omega$ is a bounded convex domain, it follows from Proposition 3.5 that for each fixed $y \in \Omega$, the function $u:=p_{t}(\cdot, y) \in W^{2,2}(\Omega)$ is the unique solution of the Neumann problem (3.5) with $f:=-\frac{d}{d t} p_{t}(\cdot, y)$. In particular, $\nabla_{x}^{2} p_{t}(x, y) \in L^{2}(\Omega)$.

Lemma 3.7. Let $\Omega$ be a bounded convex domain in $\mathbb{R}^{d}$. Then there exists $\gamma>0$ and a positive constant $C=C(\gamma)$ such that for each $y \in \Omega$,

$$
\int_{\Omega}\left|\nabla_{x}^{2} p_{t}(x, y)\right|^{2} e^{\gamma \frac{|x-y|^{2}}{t}} \mathrm{~d} x \leq C t^{-2}\left|B^{\Omega}(y ; \sqrt{t})\right|^{-1}, \quad t>0
$$

Proof. Recall that $c_{1}, c_{2}$ and $c_{5}$ are three constants appearing in (3.2), (3.3) and (3.14), respectively. Let $c_{0}=\max \left\{c_{1}, c_{2}, c_{5}\right\}$, and fix a $\gamma \in\left(0, \frac{1}{8 c_{0}}\right)$ and $y \in \Omega$. We apply Lemma 3.4 with

$$
u:=p_{t}(\cdot, y), \quad f:=-\frac{d}{d t} p_{t}(\cdot, y) \quad \text { and } \quad \psi:=e^{\gamma \frac{|\cdot-y|^{2}}{2 t}}
$$


to obtain (for some fixed $\gamma^{\prime} \in(0, \gamma)$ )

$$
\begin{aligned}
\int_{\Omega}\left|\nabla_{x}^{2} p_{t}(x, y)\right|^{2} e^{\gamma \frac{|x-y|^{2}}{t}} \mathrm{~d} x \leq & \frac{C}{t} \int_{\Omega}\left|\nabla_{x} p_{t}(x, y)\right|^{2} e^{\gamma^{\prime} \frac{|x-y|^{2}}{t}} \mathrm{~d} x \\
& +C \int_{\Omega}\left|\nabla_{x} p_{t}(x, y)\right|^{2} e^{\gamma \frac{|x-y|^{2}}{t}} \mathrm{~d} x \\
& +C \int_{\Omega}\left|\frac{\mathrm{d}}{\mathrm{d} t} p_{t}(x, y)\right|^{2} e^{\gamma \frac{|x-y|^{2}}{t}} \mathrm{~d} x \\
=: & J_{t}^{(1)}(y)+J_{t}^{(2)}(y)+J_{t}^{(3)}(y) .
\end{aligned}
$$

It follows from the gradient estimate (3.3) of $p_{t}(x, y)$ that

$$
J_{t}^{(1)}(y) \leq C t^{-2}\left|B^{\Omega}(y ; \sqrt{t})\right|^{-1} .
$$

We then use the estimate (3.14) to obtain

$$
J_{t}^{(3)}(y) \leq C t^{-2}\left|B^{\Omega}(y ; \sqrt{t})\right|^{-1} .
$$

For the term $J_{t}^{(2)}(y)$, we estimate it by considering two cases:

Case 1 . When $0<t \leq 1$, we use Lemma 3.1 to obtain

$$
\begin{aligned}
J_{t}^{(2)}(y) & \leq C \int_{\Omega} t^{-(d+1)} e^{-2 \frac{|x-y|^{2}}{c_{2} t}} e^{\gamma \frac{|x-y|^{2}}{t}} \mathrm{~d} x \\
& \leq C t^{-1}\left|B^{\Omega}(y ; \sqrt{t})\right|^{-1} \\
& \leq C t^{-2}\left|B^{\Omega}(y ; \sqrt{t})\right|^{-1} .
\end{aligned}
$$

Case 2. When $t>1$, using Lemma 3.1 again, we have

$$
J_{t}^{(2)}(y) \leq C \int_{\Omega} e^{-2 c_{3} t} e^{-2 \frac{|x-y|^{2}}{c_{2} t}} e^{\gamma \frac{|x-y|^{2}}{t}} \mathrm{~d} x \leq C t^{-2}\left|B^{\Omega}(y ; \sqrt{t})\right|^{-1},
$$

and hence, $J_{t}^{(2)}(y) \leq C t^{-2}\left|B^{\Omega}(y ; \sqrt{t})\right|^{-1}$. The desired estimate (3.15) now follows readily.

Lemma 3.8. Let $\Omega$ be a bounded convex domain in $\mathbb{R}^{d}$. There exists a constant $C>0$ independent of $y \in \Omega$ such that

$$
\int_{x \in \Omega:|x-y| \geq \sqrt{s}}\left|\nabla_{x}^{2} p_{t}(x, y)\right| \mathrm{d} x \leq C t^{-1} e^{-\gamma s /(4 t)}, \quad \forall y \in \Omega, \forall s, t>0 .
$$

Proof. Fix $s, t>0$ and $y \in \Omega$. Then using Cauchy-Schwarz's inequality and estimate (3.15) we obtain

$$
\begin{aligned}
& \int_{x \in \Omega:|x-y| \geq \sqrt{s}}\left|\nabla_{x}^{2} p_{t}(x, y)\right| \mathrm{d} x \\
& \quad \leq\left(\int_{\Omega}\left|\nabla_{x}^{2} p_{t}(x, y)\right|^{2} e^{\gamma \frac{|x-y|^{2}}{t}} \mathrm{~d} x\right)^{1 / 2}\left(\int_{|x-y| \geq \sqrt{s}} e^{-\gamma \frac{|x-y|^{2}}{t}} \mathrm{~d} x\right)^{1 / 2} \\
& \leq C t^{-1} e^{-\gamma s /(4 t)}
\end{aligned}
$$

where in the last inequality we have used the fact that

$$
\int_{x \in \Omega:|x-y| \geq \sqrt{s}} e^{-\gamma \frac{|x-y|^{2}}{t}} \mathrm{~d} x \leq C_{\gamma} e^{-\gamma s /(2 t)}\left|B^{\Omega}(y, \sqrt{t})\right|, \quad \forall s \geq 0, \quad \forall t>0 .
$$

This concludes the proof of Lemma 3.8 . 
3.2.2.3. Proof of Theorem 3.3. Let $\Omega$ be a bounded convex domain in $\mathbb{R}^{n}$. Let $\nabla^{2}$ denote generic second-order derivatives. From Proposition 3.5 it follows that the operator $T=\nabla^{2} \mathcal{L}_{N}^{-1}$ is bounded on $L^{2}(\Omega)$. Following an argument of [12], we use the formula

$$
T f=\int_{0}^{\infty} \nabla^{2} e^{-t \mathcal{L}_{N}} f d t
$$

in which the integral converges in the strong operator topology on the space of linear and bounded operators on $L^{2}(\Omega)$. Indeed, observe that

$$
\begin{aligned}
\int_{0}^{\infty} \nabla^{2} e^{-t \mathcal{L}_{N}} f d t & =\nabla^{2} \mathcal{L}_{N}^{-1} \int_{0}^{\infty} \mathcal{L}_{N} e^{-t \mathcal{L}_{N}} f d t \\
& =\nabla^{2} \mathcal{L}_{N}^{-1} \int_{0}^{\infty} \frac{d}{d t}\left(-e^{-t \mathcal{L}_{N}} f\right) d t
\end{aligned}
$$

Then use the fact that $\left\|e^{-t \mathcal{L}_{N}} f-f\right\|_{L^{2}(\Omega)} \rightarrow 0$ as $t \rightarrow 0$ and $\left\|e^{-t \mathcal{L}_{N}} f\right\|_{L^{2}(\Omega)} \rightarrow 0$ as $t \rightarrow \infty$.

It follows that the associated kernel of $T$ is given by

$$
\int_{0}^{\infty} \nabla_{x}^{2} p_{t}(x, y) d t
$$

To prove that $T$ is of weak type $(1,1)$, the idea is to make use of Theorem 3.6 above. Consider the composite operator $T\left(I-e^{-s L}\right), s>0$, whose associated integral kernel is denoted by $\tilde{K}_{s}(x, y)$. It follows that

$$
T\left(I-e^{-s \mathcal{L}_{N}}\right)=\int_{0}^{\infty}\left(\nabla^{2} e^{-t \mathcal{L}_{N}}-\nabla^{2} e^{-(s+t) \mathcal{L}_{N}}\right) \mathrm{d} t=\int_{0}^{s} \nabla^{2} e^{-t \mathcal{L}_{N}} \mathrm{~d} t,
$$

where, in the last step, we have decoupled the integrands and made a change of variables. Thus, by invoking Lemma 3.8 ,

$$
\begin{aligned}
& \int_{x \in \Omega:|x-y| \geq \sqrt{s}}\left|\tilde{K}_{s}(x, y)\right| \mathrm{d} x \leq \int_{x \in \Omega:|x-y| \geq \sqrt{s}}\left|\int_{0}^{s} \nabla^{2} p_{t}(x, y) \mathrm{d} t\right| \mathrm{d} x \\
& \quad \leq \int_{0}^{s}\left(\int_{x \in \Omega,|x-y| \geq \sqrt{s}}\left|\nabla^{2} p_{t}(x, y)\right| \mathrm{d} x\right) \mathrm{d} t \\
& \quad \leq C \int_{0}^{s} t^{-1} e^{-\gamma s / 4 t} \mathrm{~d} t \leq C^{\prime}
\end{aligned}
$$

for some finite, positive constant $C^{\prime}$ which is independent of $y \in \Omega$ and $t>0$. With this in hand, and having already established the $L^{2}$-boundedness of $T$, Theorem 3.6 applies and yields that $T$ is of weak type $(1,1)$. Hence, by interpolation, $T$ can be extended from $L^{2}(\Omega) \cap L^{p}(\Omega)$ to a bounded operator on $L^{p}(\Omega)$ for all $1<p \leq 2$.

\section{ACKNOWLEDGMENTS}

The authors would like to thank the referee for useful suggestions. The second author would like to thank X.T. Duong, S. Hofmann, D. Mitrea and M. Mitrea for helpful discussions. 


\section{REFERENCES}

[1] V. Adolfsson, D. Jerison, $L^{p}$-integrability of the second order derivatives for the Neumann problem in convex domains, Indiana Univ. Math. J. 43 (1994), 1123-1138. MR.1322613 (96e:35031)

[2] S. Agmon, A. Douglis, L. Nirenberg, Estimates near the boundary for solutions of elliptic partial differential equations satisfying general boundary conditions, (I), Comm. Pure Appl. Math. 12 (1959), 623-727. MR0125307 (23:A2610)

[3] P. Auscher, T. Coulhon, X.T. Duong, S. Hofmann, Riesz transform on manifolds and heat kernel regularity, Ann. Sci. École Norm. Sup. 37 (2004), 911-957. MR 2119242(2005k:58043)

[4] D. Bakry, M. Emery, Hypercontractivité de semi-groupes de diffusion, C. R. Acad. Sci. Paris. Sér. I Math. 299 (1984), 775-778. MR772092 (86f:60097)

[5] R.F. Bass, E. P. Hsu, The semimartingale structure of reflecting Brownian motion, Proc. Amer. Math. Soc. 108 (1990), 1007-1010. MR.1007487 (91a:60205)

[6] R.F. Bass, E. P. Hsu, Some potential theory for reflecting Brownian motion in Hölder and Lipschitz domains, Ann. Probab. 19 (1991), 486-508. MR1106272 (92i:60142)

[7] R.F. Bass, K. Burdzy, Z.-Q. Chen, Uniqueness of reflecting Brownian motion in lip domains, Ann. Inst. H. Poincaré Probab. Stat. 41 (2005), 197-235. MR2124641(2005k:60174)

[8] T. Coulhon, X.T. Duong, Riesz transforms for $1 \leq p \leq 2$, Trans. Amer. Math. Soc. 351 (1999), 1151-1169. MR.1458299 (99e:58174)

[9] T. Coulhon, A. Sikora, Gaussian heat kernel upper bounds via Phragmén-Lindelöf theorem, Proc. Lond. Math. Soc. 96 (2008), 507-544. MR2396848(2011a:35206)

[10] G. Da Prato, M. Röckner, F.-Y. Wang, Singular stochastic equations on Hilbert spaces: Harnack inequalities for their transition semigroups, J. Funct. Anal. 257 (2009), 992-1017. MR2535460(2010k:60211)

[11] E.B. Davies, Heat kernels and spectral theory, Cambridge Univ. Press, 1989. MR990239 (90e:35123)

[12] X.T. Duong, S. Hofmann, D. Mitrea, M. Mitrea, L.X. Yan, Hardy spaces and regularity for the inhomogeneous Dirichlet and Neumann problems, to appear in Rev. Mat. Iberoamericana (2011).

[13] X.T. Duong, A. Mc Intosh, Singular integral operators with non-smooth kernels on irregular domains, Rev. Mat. Iberoamericana 15 (1999), 233-265. MR1715407 (2001e:42017a)

[14] F.-Z. Gong, F.-Y. Wang, Heat kernel estimates with application to compactness of manifolds, Quart. J. Math. 52 (2001), 171-180. MR1838361(2002c:58039)

[15] A. Grigor'yan, Gaussian upper bounds for the heat kernel on arbitrary manifolds, J. Diff. Geom. 45 (1997), 33-52. MR:1443330 (98g:58167)

[16] P. Grisvard, Elliptic problems in nonsmooth domains, Pitman, Boston, MA, 1985. MR 775683 (86m:35044)

[17] P. Grisvard, G. Iooss, Problèmes aux limites unilatéraux dans des domaines non réguliers, Publications des Séminaires de Mathématiques, Université de Rennes, 9 (1975), 1-26.

[18] E. P. Hsu, Multiplicative functional for the heat equation on manifolds with boundary, Michigan Math. J. 50 (2002), 351-367. MR1914069 (2003f:58067)

[19] P. Li, S.-T. Yau, On the parabolic kernel of the Schrödinger operator, Acta Math. 156 (1986), 153-201. MR834612 (87f:58156)

[20] S. Mayboroda, M. Mitrea, Sharp estimates for Green potentials on non-smooth domains, Math. Res. Lett. 11 (2004), 481-492. MR2092902 (2005i:35059)

[21] V. Maz'ya, B. Plamenevskiǔ, Estimates in $L_{p}$ and in Hölder classes and the Miranda-Agmon maximum principle for solutions of elliptic boundary value problems in domains with singular points on the boundary, Amer. Math. Soc. Transl. 123 (1984), 1-56.

[22] D. Mitrea, M. Mitrea, L. Yan, Boundary value problems for the Laplacian in convex and semiconvex domains, J. Funct. Anal. 258 (2010), 2507-2585. MR2593333 (2010m:42049)

[23] E.M. Ouhabaz, Analysis of heat equations on domains, London Math. Soc. Monographs, Vol. 31, Princeton Univ. Press, 2005. MR2124040 (2005m:35001)

[24] M. Röckner, F.-Y. Wang, Log-Harnack inequality for stochastic differential equations in Hilbert spaces and its consequences, Infin. Dim. Anal. Quant. Probab. Relat. Top. 13 (2010), 27-37. MR2646789(2011h:60169)

[25] N. Varopoulos, L. Saloff-Coste, T. Coulhon, Analysis and geometry on groups. Cambridge Univ. Press, London, 1992. MR1218884 (95f:43008) 
[26] F.-Y. Wang, Logarithmic Sobolev inequalities on noncompact Riemannian manifolds, Probab. Theory Relat. Fields 109 (1997), 417-424. MR1481127(98i:58253)

[27] F.-Y. Wang, Gradient estimates and the first Neumann eigenvalue on manifolds with boundary, Stoch. Proc. Appl. 115 (2005), 1475-1486. MR2158015 (2006g:58054)

[28] F.-Y. Wang, Estimates of the first Neumann eigenvalue and the log-Sobolev constant on non-convex manifolds, Math. Nachr. 280 (2007), 1431-1439. MR2344874(2008m:58050)

[29] F.-Y. Wang, Semigroup properties for the second fundamental form, Docum. Math. 15 (2010), 527-543. MR2679065(2011i:58063)

[30] F.-Y. Wang, Gradient and Harnack inequalities on noncompact manifolds with boundary, Pacific Journal of Math. 245 (2010), 185-200. MR2602689 (2011b:58052)

[31] J. Wang, Global heat kernel estimates, Pacific J. Math. 178 (1997), 377-398. MR.1447421 (98g:58168)

[32] T. Yamada, S. Watanabe, On the uniqueness of solutions of stochastic differential equations, J. Math. Kyoto Univ. 11 (1971), 155-167. MR0278420 (43:4150)

School of Mathematical Sciences and Laboratory for Mathematical Complex System, Beijing Normal University, Beijing 100875, People's Republic of China - And Department of Mathematics, Swansea University, Singleton Park, SA2 8PP, United KINGDOM

E-mail address: wangfy@bnu.edu.cn

E-mail address: F.Y.Wang@swansea.ac.uk

Department of Mathematics, Sun Yat-sen (Zhongshan) University, Guangzhou, 510275, People's Republic of China

E-mail address: mcsylx@mail.sysu.edu.cn 